\title{
Conflict of Interest Disclosures
}

\section{Authors/Speakers without Conflict of Interest}

Surname, First name

Aarabi, Bizhan

Abbed, Khalid

Abdel-Wanis, Mohamed

Abdollah Zadegan, Shayan

Abduljabbar, Fahad

Abedi, Aidin

Abiola, Godwin

Abolfotouh, Sameh

Abu-Bonsrah, Nancy

Acharya, Shankar

Achilles, Felix

Adamski, Stanisław

Adjei, Joshua

Afaunov, Asker

Agrahari, Yogendra

Aguiar, Carlos

Aguilar, Tania

Aguiló, Daniela

Ahangar, Pouyan

Aharma, Pankaj

Ahmad, Alaaeldin

Ahmadje, Uzair

Ahmed, A. Karim

Ahmed, Ashfaq

Ahmed, Naeem

Ahn, Christine

Ahn, Hyo Sae

Ahuja, Christopher

Ailon, Tamir

Aiyer, Siddharth

Ajello, Marco

Ajit Kumar, N

Akbar, Muhammad

Akbulut, Deniz

Aker, Loai

Akgul, Turgut

Akinjolire, Akinwande

Akiyama, Shoshi

Akoury, Elie
Akula, Maheswara

Akula, Yeswanth

Alam, Waqar

Alant, Jacob

Albayrak, Akif

Albayrak, Kutalmış

Alberto Espitia, Carlos

Alentado, Vincent

Aleynik, Alexander

Alfayez, Saud

Alican, Mannuel Feliciano

Alini, Mauro

Alkadhaib, Bdulrahman

Alkhalid, Yasmine

Alkot, Amer

Almairac, Fabien

Almajed, Husam

Al-Mutair, Abdulaziz

Alonso, Fernando

Alotaibi, Naif

AlSaran, Yazeed

Alshafai, Nabeel

Alsultan, Omar

Altafulla, Juan

Altschul, David

Amato, Mary Kate

Amato-Watkins, Anthony

Amirjamshidi, Abbas

Ammar, Adam

Amri, Khalil

Anand, Manikant

Anandan, Kavitha

Ancheschi, Bruno

Andersen, Mikkel

Oesterheden

Anderson, Melanie

Andrade Neto, Jader

Andresen, Andreas Kiilerich

Angerler, Gerlinde
Annamalai, Devadoss

Annis, Prokopis

Aoude, Ahmed

Aranovich, Anna

Araújo Ferreira da Silva, Igor

Arce, Marysol

Arnold, Joshua

Arnold, Paul

Arnone, Gregory

Arora, Shobha

Arreola Rodríguez, Erick

Jeancarlo

Arun, Mike

Asano, Satoshi

Assietti, Roberto

Atesok, Kivanc

Athanassacopoulos, Michael

Attiah, Mark

Auerswald, Marc

Autore, Giovanni

Ávila, Luiz

Avilés, Carolina

Awwad, Waleed

Ayhan, Selim

Azadmanjir, Zahra

Aziz, Amer

Aziz, Sheweidin

Azkia, Ema Shofiana

Babak Mirzashahi, Babak

Badal, Tanya

Bader, Khaldoun

Badhiwala, Jetan

Baharudin, Azmi

Baisden, Jamie

Baklanov, Andrey

Baldew, Vyaas

Balioglu, Mehmet Bulent

Ballesteros, Vicente

Banerjee, Purnajyoti
Banta, Aditya

Bao, Hongda

Barbagallo, Giuseppe

Barbero, Andrea

Bari, Tanvir Johanning

Barimani, Bardia

Barna, Michal

Barrey, Cedric

Barry, Sean

Barsotti, Carlos Eduardo

Bartos, Marton

Barzilai, Ori

Basankin, Igor

Bashir, Muhammad Farrukh

Basiński, Krzysztof

Basso, Marco

Basu, Saumyajit

Batista, Bernardo

Batista, Marianna

Batista, Mário

Batista Jr, José Lucas

Bayram, Serkan

BC, Sureshkumar

BCM, Prasad

Becker, Christopher

Beckerman, Daniel

Beckett, Joel

Bedoya, Maria Constanza

Behr, Robert

Beland, Benjamin

Belatka, Jaroslav

Bellabarba, Carlo

Berbeo Calderon, Miguel

Enrique

Beretta, Matteo

Berg, Andrew James

Bernard, Jason

Bernd, Wegener

Berry, Abigale 


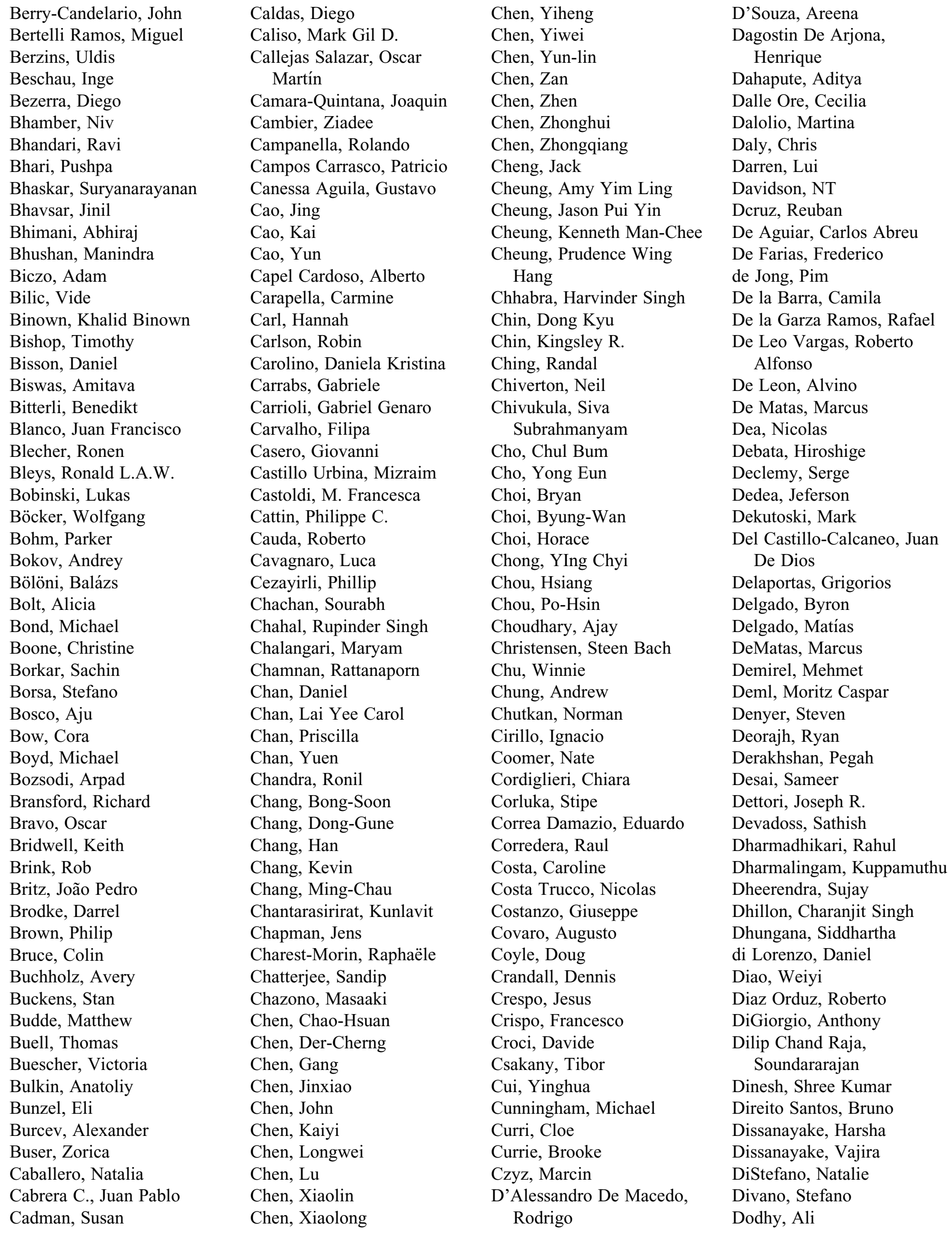


Dogar, Ammar

Dokuzovic, Stjepan

Domenicucci, Maurizio

Domínguez, Maria Teresa

Donato Amorim, Gabriel

D'Oro, Anthony

Doze, Karlis

Dozza, Diego Cassol

Drazin, Doniel

Driscoll, Adam

Du, Yueqi

Duan, Pingguo

Duan, Wanru

Duan, Wan-Ru

Duarte, Rui

Ducati, Alessandro

Duff, John

Dumpa, Srikant Reddy

Dunn, Colin

Dusad, Tarun

Dutta, Shumayou

Dvorak, Marcel

Dwivedi, Rishi

Dzhafarov, Vidzhay

Ealing, John

Echt, Murray

Eder, Claudia

Eglin, David

Eisa, Amr

El-Adawy, Amr

ElHewala, Tarek

Eltes, Peter

Endo, Teruaki

Eösze, Zsolt

Eppinga, Wietse S C

Epure, Laura

Ernst, Carsten

Esfahani, Darian

Esteves Simões, Christiano

Eum, JinHwa

Evsukov, Aleksey

Evsyukov, Alexey

Faghih, Morteza

Fairag, Rayan

Falavigna, Asdrubal

Fantoni, Massimo

Faraj, Khaled

Faraj, Sayf

Farsetti, Pasquale

Faruk Senan, Nur Aida

Faschingbauer, Maximilian

Feijó Scharf, William

Felipe Lauf Melotti, Arthur

Felli, Lamberto

Feng, Ruiqing

Fenyöhazi, Eszter
Ferguson, Stephen J.

Ferland, Catherine E.

Fernando, Alonso

Ferreira, Ricardo

Filatov, Egor

Fimian, Chantal

Finkelstein, Anna

Finkelstein, Joel

Fisahn, Christian

Fischer, Charla

Fleiderman, José

Fong, Angus

Formica, Carlo

Formica, Matteo

Foster, Mitchell

France, John

Fueki, Keisuke

Fuentealba Contreras, Boris

Fuentes Rivera, Miguel Ángel

Fuji, Takeshi

Fulcher, Alex

Fulzele, Sadanand

Furbino de Pinho Valentim,

Larissa

G I, Siddalingeshwara

Gadde, Saikrishna

Gaete, Cesar

Gajudo, Gio

Galina, Jesse

Galiner-Ron, Masha

Gandhi, Shashank

Ganesan, Sudhir

Gaonkar, Bilwaj

Garbossa, Diego

Garcia, Maria Alejandra

Garcia Ortiz, Uriel Nahum

Garcia-Medrano, Belen

Garfinkel, Ignacio Gabriel

Gargent, Sara

Gati, András

Gautam, Sai

Gavassi, Bruno

Gay, Max

Gelfand, Yaroslav

George, Joshi

George, Michelle

Germscheid, Niccole

Gerochi, Renier D.

Geurts, Jeroen

Ghaffar, Samia

Ghanem, Ahmad

Ghodsi, Zahra

Ghogawala, Zoher

Gibson, Alexander

Gielis, Willem Paul

Gimbel, David
Gimenez-Gigon,

Maximiliano

Giovannetti, Maddalena

Glennie, R. Andrew

Go, Ban Suk

Godoy, Wilmer

Goh, Graham Seow-Hng

Goh, Jeremy

Goh, Keng Meng Jeremy

Golan, Jeff

Golubovsky, Joshua

Gomez, Jonathan

Gomez Cristancho, David Camilo

Gonçalves Brasil Neto, Coracy

Goncalves Paris, Louise

Gonschorek, Oliver

González, Oscar

González Bustamante, Catalina

González Moga, Amado

Goodrich, James

Goon, Kelsey

Gorgoglione, Nicola

Gould, Heath

Goz, Vadim

Grad, Sibylle

Grant, Michael

Greiner, Axel

Greutert, Helen

Grevitt, Michael

Griessen, Mathias

Griffith, James Francis

Grimm, Dulce

Grossman, Robert

Grützner, Paul A.

$\mathrm{Gu}$, YuTong

Gualtierotti, Roberta

Guan, Li

Guarnaccia, Laura

Gubin, Alexander

Guerard, Karl-Philippe

Guha, Daipayan

Guiloff, Benjamin

Gunness, Nitish

Guo, Yan

Guo, Zhaoqing

Gupta, Anshul

Gupta, L N

Gupta, Pravin

Gurung, Ganesh

Guzman, Raphael

Guzman Carranza, Juan

Enrique

Haanstra, Tsjitske
Habib, Eva

Haglund, Lisbet

Hahne, Julia

Hai, Yong

Hailong, Feng

Haines, Colin

Hajnovic, Ludovit

Haleem, Meraaj

Hall, Hamilton

Hallager, Dennis Winge

Hanbali, Yahia

Hanif, Asif

Hansen, Lars Valentin

Haraluru Jayanna, Harsha

Harman, Clare

Harrop, James

Hartmann, Sebastian

Hasan, Ghazwan A.

Hasan, Nahla M

Hasharoni, Amir

Hashmi, Sohaib

Hattori, Gohsuke

Hauck, Stefan

Hausmann, Oliver N.

Heesterbeek, Petra

Heider, Franziska

Heravi, Amir

Hermida, Jose

Hernandez, Gilmar Leonardo

Hernandez, Laura

Hernandez, R. Nick

Hernandez Cruz, Angel

Hernandez-Ramajo, Ruben

Herrera, Cristhian

Herrero, Carlos Fernando

Hersey, Denise

Hershkovich, Oded

Herzog, Jan

Hida, Kazutoshi

Hiller, Nurith

Hirose, Tomohiko

Hitzl, Wolfgang

Hoel, Ryan

Hoffman, Eve

Hogue, Olivia

Hohenberger, Gloria Maria

Holewijn, Roderick M.

Holmes, Laura

Holton, James

Homans, Jelle

Hong, Jae Taek

Hong, James

Hoshino, Yushi

Houkin, Kiyohiro

Houri, Saadit Sarah

Howard, Rachel 


\begin{tabular}{|c|c|}
\hline $\mathrm{Hu}$, Bowen & Jenkin, Graham \\
\hline $\mathrm{Hu}$, Emily & Jenner, Edward \\
\hline Huang, Hsiang-Ming & Jensen, Cyrus \\
\hline Huang, Ting Chun & Jeong, Soyeon \\
\hline Huber, Gerd & Jespersen, Annette \\
\hline Hulme, Chris & Bennedsgaard \\
\hline Humadi, Ali & Jezieniecki, Carlos \\
\hline Hur, Jung-Woo & $\mathrm{Ji}$, Hongquan \\
\hline Hussain, Haseeb & Jian, Fengzeng \\
\hline Hussain, Ibrahim & Jiang, Enze \\
\hline Hustedt, Joshua & Jiang, Fan \\
\hline Iacovazzi, Davide & Jiang, Shuai \\
\hline Ibrahim, George & Jiang, Wei-yu \\
\hline Ibrahim, John & Jiang, Yu \\
\hline Ibrahim, Kamalnizat & Jinping, Liu \\
\hline Iencean, Andrei Stefan & Jiwani, Zaneen \\
\hline Iencean, Stefan Mircea & Jjhala, Amit \\
\hline Ifesemen, Onosi S. & Jo, Hyunjin \\
\hline Iizuka, Takahiro & Jo, Hyun-Jin \\
\hline Ilyas, Haariss & John, Jobin \\
\hline Im, Gi Hye & Jones, Morgan \\
\hline Imsdahl, Sheri & Jorge Fontoura Solla, Davi \\
\hline Inagaki, Katunori & Jost, Gregory F. \\
\hline Ino, Masatake & Jung, Dong-Wook \\
\hline Inoue, Hirokazu & Jung, Heung-Tae \\
\hline Insinga, Salvatore & Jung, Yong Tae \\
\hline Isa, Mohammed & K L, Kalra \\
\hline Islam, Ariful & Kailash, Karthik \\
\hline Ito, Manabu & Kale, Shashank \\
\hline Itoh, Kanako & Kalinosky, Benjamin \\
\hline Itshayek, Eyal & Kalsi-Ryan, Sukhvinder \\
\hline Iundusi, Riccardo & Kamenova, Maria \\
\hline Iutaka, Alexandre & Kamysheva, Valentina \\
\hline Ivanov, Marcel & Kanai, Masayoshi \\
\hline Iwami, Toyohito & Kandwal, Pankaj \\
\hline Iwanaga, Joe & Kang, Jong-Won \\
\hline Iwasaki, Motoki & Kang, TaeWook \\
\hline Izquierdo, Guillermo & Kanna, Rishi Mugesh \\
\hline Jacob Jr, Charbel & Kannan, Abhishek \\
\hline Jada, Ajit & Kanz, Karl Georg \\
\hline Jain, Mantu & Kaplan, Leon \\
\hline Jain, Nick & Kapoor, Vik \\
\hline Jaja, Blessing & Karabatsou, Tina \\
\hline Jakkan, mithun & Karadimas, Spyridon \\
\hline Jakubovic, Raphael & Kargin, Deniz \\
\hline Jakus, Adam & Karim, Farhan \\
\hline Jalgaonkar, Azal & Karunanayake, Aranjan \\
\hline Janssen, Insa & Kashiro, Atsuji \\
\hline Jansson, Volkmar & Kasis, Ata \\
\hline Jarzem, Peter & Kasperts, Nicolien \\
\hline Javed, Shahzad & Katchko, Karina \\
\hline Quillo-Olvera, Javier & Kato, So \\
\hline Jayabalan, Vignesh & Kaushal, Mayank \\
\hline Jazayeri, Seyed Behnam & Kawahara, Norio \\
\hline Jazayeri, Seyed Behzad & Kawakami, Mamoru \\
\hline Jefferson, Wilson & Kaye, Angus \\
\hline
\end{tabular}

Kaygusuz, Mehmet Akif

Kebaish, Khaled

Keijsers, Noël L.W.

Kelkar, Amey

Keller, Leonard

Kellner, Christoph

Kelmakov, Vladislav

Kerekes, Daniel

Kesharwani, Sandeep

Kha, Stephanie

Khalepa, Roman

Khazaei, Mohamad

Khazaie, Zahra

Kheirkhah, Pouyan

Khoury, Amal

Khoyratty, Sara

Kiapour, Ali

Kikkawa, Ichiro

Kim, Eliana

Kim, Eliana Eunhee

Kim, Ho-Joong

Kim, Il Sup

Kim, In-Bo

Kim, Jin-Hyok

Kim, Keun Su

Kim, Kyung Hyun

Kim, Rachel

Kim, Sung Kyu

Kim, Tae JIN

Kim, Woo Kyung

Kim, Yong Min

Kimura, Atsushi

Kimura, Tomoatsu

Kingma, Idsart

Kirat, Akay

Kirzner, Nathan

Kishor, Hari

Kiss, Laszlo

Kitajima, Isao

Kitson, Jonathon

Klimov, Vladimir

Kloc, Wojciech

Kobata, Kim

Kobayashi, Kenji

Kobets, Andrew

Kocak, Tugrul

Kodigudla, Manoj

Koff, Marco Antonio

Koh, Hui Yu

Kokabu, Terufumi

Kolb, Luis

Kolesov, Sergey

Kondrashov, Dimitriy

Konishi, Hiroaki

Konovalov, Nikolay

Korkmaz, Murat
Korolishin, Vasiliy

Kozelnicka, Kristina

Krassnig, Renate

Kraus, Michael

Kreinest, Michael

Krik, Alexandr

Krisno, Novan

Krock, Emerson

Kudo, Yoshifumi

Kuh, Sung Uk

Kulkarni, Arvind

Kumagae, Yoshio

Kumar, Jay

Kumar, Naresh

Kumar, Prabhat

Kundnani, Vishal

Kunej, Tomi

Kunieda, Takeharu

Kuperus, Jonneke

Kurlandt, Patryk

Kutlaeva, Marina

Kuzmenko, Alexandr

Kwan, Kenny Yat Hong

Kwan, Kevin

Kwok, Kin On

Kwon, Brian

La Rosa, Guido

Lacroix, Michel

Lafage, Renaud

Lai, Maksim

Laloux, Etienne

Lam, Khai

Lama, Polly

Lamas, Vincent

Landowski, Peter

Lang, Gernot

Langlais, Tristan

Lapaeva, Olga

Lapointe, Jacques

Laratta, Joseph

Lattig, Friederike

Lau, Lok Ting

Lau, Yvonne Yan On

Law, Annie

Law, Sheung Wai

Lazary, Aron

Leary, Emily

Leasure, Jeremi

Lecaros, Javier

Lee, Bryan

Lee, Choon-Ki

Lee, Han-Chung

Lee, Jong Beom

Lee, Jung Jae

Lee, Jung-Hee

Lee, Ka Lok Ryan 


\author{
Lee, Kee Chang \\ Lee, Sang Gu \\ Lee, Wendy XP \\ Lee, Wen-Shen \\ Leidinger, Andreas \\ Lener, Sara \\ Lertudomphonwanit, \\ Thamrong \\ Leschke, Jack \\ Levchenko, Evgeniy \\ Lewis, Stephen \\ Li, Guangzhou \\ Li, Jia Xi Julian \\ Li, Quan You \\ Li, Shi-Jiang \\ Li, Tao \\ Li, Tuo \\ Li, Wei \\ Li, Wei Shi \\ Li, Weishi \\ Li, Wenjun \\ Li, Yue \\ Li, Yueying \\ Lian, Xiaofeng \\ Liang, Bo \\ Liao, Shiyao \\ Libionka, Witold \\ Licci, Maria \\ Liebergall, Meir \\ Liew, Susan \\ Lim, Paul \\ Lim, Tae Kyoo \\ Lin, Guang-Xun \\ Lin, Hsi-Hsien \\ Lin, James \\ Line, Bret \\ Ling, Marcus \\ Ling, Zhixing Marcus \\ Link, Thomas \\ Linninger, Andreas \\ Liow, Lincoln \\ Liu, Hao \\ Liu, Jingwei \\ Liu, Limin \\ Liu, Shunan \\ Liu, Tie \\ Liu, Yilin \\ Liu, Yuzeng \\ Liu, Zhen \\ Liu, Zhenlei \\ Liu, Zhuojie \\ Lo, Haoju \\ Locatelli, Marco \\ Lombardi, Joseph \\ Lonner, Baron \\ Loo, Wee Lim
}

Loparev, Evgeniy

Lou, Zijian

Lubbe, Ryan

Lubinski, Jake

Lui, Darren

Luís, Ana

Luk, Dip-Kei Keith

Luna, Jeffrey

Luna A., Francisco

Lv, Yang

Lyu, Qiunan

M L V, Sai Krishna

Ma, Wei-Hu

Maccauro, Giulio

Machado Cardoso, Igor

Macyszyn, Luke

Mafra, Inês

Magalhães Menezes, Cristiano

Magomedov, Shamil

Maheswaran, Anupama

Maia, Thiago

Majoran, Tom

Makino, Hiroto

Malahov, Sergey

Maling, Lucy

Malley, Michael

Malloy IV, John

Mamaril, Lulu Joan

Manabe, Nodoka

Mancini, Federico

Mandal, Subhodeep

Mann, Koren

Manolarakis, Georgios

Mansouri, Alireza

Mao, Saihu

Maranho, Daniel

Marengo, Nicola

Marfia, Giovanni

Mariani, Luigi

Marítnez, Carlos

Marqués, Carlos

Marré, Bartolome

Martens, Frederic

Martin, Allan

Martin, Brook I.

Martin, Christopher

Martin, Ivan

Martínez, Celmira

Martinez - Anda, Jaime Jesus

Maruyama, Hiroshi

Maryam, Farah

Masevnin, Sergey

Mashfiqul Arafin Siddiqui, Mohammad

Massicotte, Eric
Masuda, Koici

Matejka, Jiri

Matejka, Tomas

Matera, Julia A.

Mathur, Vineesh

Matschke, Stefan

Matsui, Seishi

Matsumoto, Koji

Matsuyama, Yukihiro

Matsuzawa, Ryo

Mawdsley, Matthew

May, Karen

Mayadev, Angeli

Mayr, Matthias

McAnany, Steve

McCall, Iain

McClendon, Mark

Mcgonagle, Lorcan

McGowan, Mark

McGwin, Gerald

McLaughlin, Lily

Medina Barra, Luis

Medvedeva, Svetlana

Mehdi, Syed

Mehdian, Hossein

Mehrkens, Arne

Mehta, Ankit

Mehta, Gaurav

Mehta, Vikram

Mejia Munne, Juan Carlos

Melcher, Carolin

Meldau, Jason

Meneses, David

Meng, Tong

Menon, Jagdish

Merello, Bernardo

Meric, Emre

Mert, Muhammed

Messer, Philipp

Metzger, Peter

Mewara, Navin

Meyer, Bernhard

Michailov, Dmitry

Migalkin, Nikolay

Milan, Kate Louise

Milavec, Helena Maria

Millecamps, Magali

Mindedahl Jespersen, Stig

Mine, Ken

Mirabile, Belin

Miracle Nayagam, Sharon

Miranda, Saulo

Miranda Linhares, Kleber

Mirbazegh, Fatemeh

Mirchev, Nikolay

Miscusi, Massimo
Mishagin, Alexey

Mistry, Manish

Mitchell, Sean

Miyamoto, Hiroshi

Miyazaki, Ryohei

Mlyavykh, Sergey

Moammer, Gemeh

Mobbs, Ralph

Modahi, Nawaf

Moghaddamjou, Ali

Mohamed, Mohamed

Mohamed Ramlee, Fadzrul Abbas

Mohammadzadeh, Mahdi

Mohd Amin, Mohamad Zaki

Mokawem, Michael

Molina Salinas, Marcelo

Montejo, Julio

Montero, Carlos

Moon, So Hyun

Moore, Don

Mora Ávila, René Crisanto

Morales, Luis Carlos

Morgado Vázquez, Jonathan Samuel

Morgan, Clare

Morioka, Motohiro

Morlock, Michael M.

Morris, Matthew T.

Mostert, Adriaan K.

Movrin, Igor

Msuya, Salim Rashidi

Muhamad Ariffin, Mohd Hisam

Muller Ávila, Luiz

Munhoz da Rocha, Luís Eduardo

Muni, Saurabh

Munigangaiah, Sudarshan

Munk, Helle

Muñoz - Romero, Ildefonso

Muñoz C., Rubén

Munroe, Brent

Munteanu, Alex

Muntion, Sandra

Muqeet, Vaishnavi

Murata, Hidetoshi

Murawski, Piotr

Mushkin, Alexandr

Mushkin, Mikhail

Musmar, Haytham

Muthukumar, Natarajan

Muthurajan, Raveendran

Mutschler, Wolf

Nabiyev, Vugar

Nadeem, Umair 
Nagashima, Hideki

Naghdi, Khatereh

Nakahara, Shinnosuke

Nakamura, Taishi

Nakamura, Yutaka

Nakano, Masato

Nakhla, Jonathan

Naresh-Babu, J

Nash, Alysa

Nashikar, Piyush

Nasiri Bonaki, Hirbod

Nasser, Rani

Nassiri, Farshad

Nasto, Luigi

Nater, Anick

Naumenko, Zinaida

Naumov, Denis

Navaladi Shankar, A

Navarro-Ramirez, Rodrigo

Navone, Stefania Elena

Neese, Ashley

Nelson, Sandra B.

Nemirovsky, Carlos Eduardo

Nesnidal, Petr

Nesterenko, Pavel

Netzer, Cordula

Nguyen, Quynh

Nicholls, Fred

Nicholls, Kate

Nicoli, Daniele

Niedzielak, Tim

Nielsen, Christopher J.

Niemeier, Thomas

Nirino, Carlos Mariano

Niu, Shuo

Niu, Tianyi

Nizzoli, Vitaliano

Nogueira-Barbosa, Marcello

Noh, Sung Hyun

Norvell, Daniel

Nouisri, Lotfi

Novo Brazolino, Marcus

Alexandre

Novoa, Felipe

Nowacki, Amy

Ochirova, Poleena

Odeh, Khalid

ODonnell, Jeffrey

Oehme, David

Oggiano, Leonardo

Ogilvie, Shahiba

Oh, Kevin

Oh, Yoong Leong Jacob

Ohiorhenuan, Ifije

Ohori, Yasuo

Ohtori, Seiji
Oitment, Colby

Olivares Camacho, Jorge Luis

Olivares Peña, Jorge Luis

Oliveira Bretas, Caroline

Ollero, Lourdes

Olynnger, Tom

Öner, Ali

Oni-Orisan, Akinwunmi

Ono, Masatoshi

Oral, Ismet

Oshina, Masahito

Oskouian, Rod

Osmani, Humza

Osterheden Andersen, Mikkel

Overgaard, Soeren

Owen, Michael

Oxner, William

$\mathrm{P}$, Srinath

Pacca, Paolo

Pahapill, Richard

Paholpak, Permsak

Pahuta, Markian

Palaoglu, Selcuk

Palmer, Joseph

Palmer Becerra, Romeo

Palukuri, Nagendra

Pambianco, Virginia

Pan, Aixing

Pan, Zhimin

Pandey, Prabhat

Pandita, Naveen

Pang, Chuan

Pankowski, Rafał

Pantoja Contreras, Samuel

Paolicelli, Michelle

Papastefan, Steven

Paquette, Scott

Paquis, Philippe

Parikh, Nisarg

Park, Eric Taejin

Park, Jeong Yoon

Park, Jiwon

Park, Jong-Beom

Park, SiYoung

Pasku, Dani

Patel, Ankit

Patel, Kishan

Patel, Nimesh

Patel, Pavan

Patel, Pranay

Patel, Ravish

Patil, Nirmal

Pattnaik, Ashish

Paudel, Santosh

Paulus, Alexander

Pavlov, Orlin
Pavlova, Olga

Pearce, Kalin

Pecache, Monica

Pecoraro, Michele Federico

Pedreira, Rachel

Pehlivanoglu, Tuna

Pelle, Dominic

Penner, Federica

Pennes, Florent

Pennington, Zachary

Perera, Romain

Perez Rodriguez, Juan Carlos

Peroglio, Marianna

Perry, Tony

Persohn, Sylvain

Pescador, David

Petrie, Michael

Petrone, Salvatore

Phan, Kevin

Philippens, Marielle E P

Pillay, Robin

Pinto Coelho Fontes, Bruno

Pithwa, Yogesh

Plaza Wuthrich, Sonia

Pluderi, Mauro

Plyasov, Sergey

Pola, Enrico

Polly, David

Pompili, Alfredo

Popa, Radu

Popp, Erik

Porhanov, Vladimir

Poubel Bolelli de Rezende, Rafael

Pouran, Behdad

Prada Solano, Stefania

Prakash, Rahul

Pratali, Raphael

Prins, Gail

Prudnikova, Oxana

Ptashnikov, Dmitrii

Puchwein, Paul

Pueschel, Klaus

Puhl, Maria

Punyarat, Prachya

Purviance, Connor

Purvis, Taylor

Pyo, Se Young

Qi, Qiang

Qian, Bangping

Qiao, $\mathrm{Mu}$

Qiu, Yong

Quadros, Francine

Quillo-Olvera, Javier

Quinn, John

Quintanilla, Marcela
Quisth, Lena

Quraishi, Nasir

Qutteineh, Bilal

Raasck, Kyle

Racek, Jaroslav

Racine, Linda

Racinowski, Maciej

Raco, Antonino

Rae Zubkov, Micaella

Rafrafi, Abderrazek

Rai, Alok

Rai, Anil Kumar

Raina, Prince

Rajamani, Vijay

Rajaram, Sakthivel

Rajasekaran,

Shanmuganathan

Ramieri, Alessandro

Ramos, Miguel Rafael

Rampersaud, Y. Raja

Rampini, Paolo

Rangel Morales, Carlos Raul

Ranjan, Alok

Rasteryaeva, Marina

Ravikumar, Nivetha

Ravinski, Robert

Reed, Ryder

Reese, Jared C.

Rehousek, Petr

Reichel, Heiko

Reiner, Anne

Reizinho, Carla

Rekate, Harold

Rendón, Diego

Rho, Young Joon

Riabykh, Sergey

Ribeiro, Elisabete

Riboni, Laura

Ricciardi, Daniel Oscar

Ricciardi, Guillermo

Alejandro

Rienmüller, Anna

Righesso, Orlando

Rivas, Walter

Robinson, Yohan

Rocha, Luis

Rocławski, Marek

Ronai, Marton

Roncetti Junior, Ronaldo

Rose, Peter

Rosenzweig, Derek

Rosero, Diana

Roßbach, Björn

Rothlauf, Paulina

Roughley, Peter

Rozo, Gustavo 


\begin{tabular}{|c|c|}
\hline Rubenbauer, Bianka & Schneider, Andrew \\
\hline Ruini, Marco & Schneider, Julia \\
\hline Ruiz, José & Schneider, Niko R. E. \\
\hline Ruparel, Sameer & Schreyer, Sylvia \\
\hline Rushton, Paul R. P. & Schroeder, Josh \\
\hline Russo, Salvatore & Schroeder, Joshua \\
\hline Rustagi, Tarush Rustagi & Schulthais da Silva, Flavia \\
\hline Ryabykh, Sergey & Schulz, Jacob F \\
\hline Rychen, Jonathan & Schuster, James \\
\hline Ryu, Kyeong-Sik & Schütz, Ludwig \\
\hline Sá Barros, Cecília & Schwab, Joseph \\
\hline Saadi, Saber & Sedia, Mattia \\
\hline Saavedra Palma, Tatiana & Seffen, Thomas \\
\hline Sabalic, Srecko & Sefranek, Vladimir \\
\hline Sadeghian, Farideh & Segovia Mera, Ramon \\
\hline Sadeh, Morteza & Seibert, Fanz Josef \\
\hline Sadowska, Aleksandra & Seki, Shoji \\
\hline Safaee, Michael & Seki, Toshitaka \\
\hline Safdarian, Mahdi & Seksenyan, Akop \\
\hline Saghal, Arjun & Sellenschloh, Kay \\
\hline Sagor, M.Asraf Ul Matin & Selvadurai, Susanne \\
\hline Sahin, Koray & Sembrano, Jonathan \\
\hline Sahin, Yasin & Senan, Nur Aida F \\
\hline Saifutdinov, Marat & Senarath, Upul \\
\hline Salim, Azizul Akram & Seng, Wei Ren Daniel \\
\hline Samala, Raghu & Sessa, Giovanni \\
\hline Samartzis, Dino & Sessa, Sergio \\
\hline Samsoer, Lima & Setiobudy, Tony \\
\hline Sánchez-Guijo, Fermín & Seyed Vosoughi, Ardalan \\
\hline Saniville, Philip & Shabani, Hamisi K. \\
\hline Sankey, Eric W. & Shackleford, Ian \\
\hline Santoro, Giorgio & Shah, Akash A. \\
\hline Santos, Alexandra & Shah, Anoli \\
\hline Santos Benitez, Hugo Alberto & Shah, Ishan \\
\hline Santos Franco, Jorge Arturo & Shah, Ramille \\
\hline Sapare, Sarang & Shankar, Ganesh \\
\hline Sar, Cuneyt & Shapovalov, Vladimir \\
\hline Saran, Neil & Sharif-Alhoseini, Mahdi \\
\hline Saravankumar, Gurunathan & Sharma, AK \\
\hline Sarkar, Mehul & Sharma, Akshay \\
\hline Sasaki, Kanji & Sharma, Ayush \\
\hline Sassi, Marco & Sharma, Deep \\
\hline Sato, Atushi & Sharma, Rajesh Kumar \\
\hline Sato, Mitsuru & Sharma, Ravi \\
\hline Sauer, Daniel & Shawk, Ahmad \\
\hline Savarirajo, Jude Cornelius & Shear Yashuv, Hananel \\
\hline Savin, Dmitry & Shen, Cailiang \\
\hline Schaeren, Stefan & Sher, Idrees \\
\hline Schildböck, Sabrina & Sher, Mustafa \\
\hline Schildhauer, Thomas A. & Shetty, Ajoy \\
\hline Schirinzi, Antonio & Shi, Benlong \\
\hline Schlösser, Tom & Shiban, Ehab \\
\hline Schmidt, Cameron & Shiban, Youssef \\
\hline Schmidt Brock, Roger & Shigekawa, Seiji \\
\hline Schmiesing, Andrew & Shigematsu, Hideki \\
\hline Schmit, Brian & Shillingford, Jamal \\
\hline
\end{tabular}

Shimizu, Takachika

Shimmon, Ronald

Shin, John

Shinde, Sushil kumar

Shinohara, Kensuke

Shirahata, Toshiyuki

Shiraishi, Yasuyuki

Sia, Ung

Siddiqui, Shiblee S

Sides, Brenda

Silva, Alvaro

Silva, Keli

Simoes, Leonardo

Simony, Ane

Singh, Gurmit

Singh, Jagdeep

Singh, Mahendra

Singh, Saurabh

Singh, Sunit

Singh, Vijay

Sinigaglia, Aldo

Sinkemani, Arjun

Skalli, Wafa

Sloan, Stephen

Slugin, Evgeny

Smit, Esther

Smith, Julian

Smith, Mike

Smith, Walter

Soccol, Fernando

Soh, Calvin

Soh, Reuben

Solanki, Anil

Soleman, Jehuda

Sommaruga, Samuel

Son, Seung

Sonawane, Dhiraj

Song, Dianwen

Song, Ruipeng

Song, Yueming

Sonone, Sandeep

Soriano-Sanchez, JoseAntonio

Soriano-Solis, Sergio

Soroka, Avraham

Souza, Camila

Sovpenchuk, Igor

Sperduti, Isabella

Spina, Nicholas

Šprem, Jurica

Spruit, Maarten

Squires, Mathieu D.

Sri Kumar, Sudhir Kumar

Srinivas, Shreya

Srivastava, Sudhir

Stadhouder, Agnita
Stark, Michael

Steadman, Tobias

Steverink, Jasper G

Stieber, Jonathan

Stock, Stuart

Stokes, Oliver

Stone, Laura

Strantzas, Samuel

Street, John

Stricker, Sarah

Stulik, Jan

Stupp, Samuel

Sturzeneker Cerqueira Lima, Ricardo

Subagio, Eko

Subbiah, Jayakumar

Sudershan, Sushil

Sudo, Azusa

Sugawara, Ryo

Suh, Seung-Woo

Suk, Se-Il

Sun, Chuiguo

Sun, Haolin

Sun, Woo-Seong

Sun, Xiangyao

Sun, Xin

SUN, Xu

Sun, Yu

Sun, Zhuo Ran

Sunderland, Geriant

Sung, Jaehoon

Sureja, Kelvin

Suresh, Gayathri

Suzuki, Kayo

Szollosi, Balazs

Szövérfi, Zsolt

Taboada, Nestor

Tahmazyan, Karapet

Takai, Tetsuro

Takeshita, Kastushi

Takeuchi, Kazuhiro

Tamai, Koji

Tan, Barry

Tan, Boon Beng

Tan, Gladys P.

Tan, Hooi Ming

Tan, Jonathan

Tan, Lee

Tan, Seang Beng

Tan, Tiam Siong

Tanaka, Takaaki

Tanaka, Takahiro

Tanenbaum, Joseph

Tang, Yale

Tangavel, Chitraa

Tanouchi, Testu 
Tao, Hui

Tarantino, Umberto

Tarpada, Sandip P.

Tatarintsev, Alekandr

Tateishi, Kensuke

Taylor, Ben

Tee, Jin

Teixeira Jacobsen, Manoel

Telera, Stefano

Teles, Alisson

Teo, Terry

Teo, Yian Young

Terayama, Sei

Tetreault, Lindsay

Thambiraj, Sathya

Theiss, Steven

Thibaut, Carlos

Thomas, Kenneth

Thumbadoo, Ruben

Tian, Yun

Tilkema, Thomas D.

Timothy, Bryan

Tlemsani, Karim

To, Michael

Todd, James

Toh, Nicole XL

Tomak, Patrick

Tomina, Marina

Tomita, Kazunari

Tomlinson, James

Tøndevold, Niklas

Torche V., Esteban

Torche V., Máximo

Torrens, Juan Pablo

Touli, Ermioni

Tounsi, Ahmed

Toyone, Tomoaki

Tresserras Gine, Gloria

Tretyakova, Anastasiay

Triparthy, Sujit

Tristancho, Alexander

Trivedi, JM

Tromp, Isabel

Tschugg, Anja

Tsiouris, Apostolos

Tubbs, R. Shane

Tuchman, Alex

Uchikado, Hisaaki

Ujjan, Badar

Ukunda, Fred U N

Umenushkina, Ekaterina

Urbansky, Milan

Uvaraj, Nalli Ramanathan

Uz Zaman, Atiq

V, Ravi

Vaccaro, Alexander
Valdés I., Guillermo

Valencia, Javiera

Valencia, Manuel

Vallabh, Sagar

Van Beaver, Laura

van Hamersvelt, Robbert

van Herwaarden, Joost

van Hooff, Miranda

van Solinge, Guido B.

van Tol, Floris

Varanda, Pedro

Vargas, Victoria

Vasilenko, Ivan

Vasquez, Luis Jesus

Vastmans, Jan

Vela, Viviana

Velasquez, Gina

Velez, Camilo

Velnar, Tomaz

Venkatesan, Muralidharan

Ver, Mario

Vercelli, Giovanni

Veres, Robert

Verlaan, Joannes J

Verlaan, Jorrit-Jan

Verma, Swetabh

Veroni, Andrea

Versace, Alessandro

Vialle, Luiz Roberto

Vigueras A., Sebastián

Villaroman, Diane

Vint, Helen

Viola, Árpád

Virk, Michael

Virojanapa, Justin

Viswanadha, Arun-Kumar

Volinnskiy, Alexey

Volquind, Daniel

Vorsic, Matjaz

VVR, Chandra

Wahlefeld, Jan

Waldrop, Robert

Walker, Brett

Wan Ab Rahman, Wan Zainuddin

Wang, Bin

Wang, Christopher

Wang, Dechun

Wang, Feng

Wang, Jingming

Wang, Lei

Wang, Limin

Wang, Linnan

Wang, Patrick

Wang, Qing

Wang, Samuel
Wang, Shih-Tien

Wang, Song

Wang, Weidong

Wang, Yan

Wang, Yuqiang

Wanke-Jellinek, Lorenz

Wanyoike, Peter

Waqar, Mueez

Watanabe, Hideaki

Weber, Michael

Weckbach, Sebastian

Wegener, Bernd

Wei, Jianwei

Weil, Yoram

Weiss, Thomas

Wendolowski, Stephen

Whicker, Emily

White, Melissa

White, Timothy

Wiechert, Karsten

Wijayaratne, Lalith

Wilkening, Matthew

Willems, Stefan M

William, Yeo

Williams, Jack

Wilson, Jefferson

Wilson, Robert

Wipplinger, Christoph

Wiśniewski, Jakub

Witiw, Christopher

Wolinsky, Jean-Paul

Won, Yougun

Wong, Carlos King Ho

Wong, Chung Chek

Woo, Youngha

Woodacre, Timothy

Wright, Anna

$\mathrm{Wu}$, Xiao-Tao

Wuertz-Kozak, Karin

Xia, Chao

Xia, Zhiyuan

Xie, Lin

Xie, Zhi-Yang

Xin, Zhijun

$\mathrm{Xu}$, Jianguang

$\mathrm{Xu}$, Leilei

$\mathrm{Xu}$, Risheng

$\mathrm{Xu}$, Sheng

Yadav, Gagandeep

Yahara, Yasuhito

Yaldo, Omar

Yamada, Hiroshi

Yamamoto, Tetsuya

Yamasaki, Ryoji

Yamazaki, Kazuyoshi

Yan, Peng
Yanamadala, Vijay

Yang, Hao

Yang, Huiliang

Yang, Jae Hyuk

Yang, Qingguo

Yang, Seung-Ho

Yang, Xi

Yao, Yao

Yao, Yu-Cheng

Yasuda, Taketoshi

Yasui, Keigo

Yaszay, Burt

Ye, Diana-Luk

Yee, Albert

Yee, Phillina

Yeo, William

Yeom, Jin Sup

Yiglor, Calgar

Yilmaz, Emre

Yin, Huabin

Yiu, Karen

Yoganandan, Narayan

Yong, $\mathrm{Hu}$

Yoon, Wai Weng

Yoshimizu, Takaki

You, Eunice Linh

Yousef, Mutaz

Yson, Sharon

$\mathrm{Yu}$, Hai Ming

YU, Yang

Yue, Wai Mun

Yuksel, Selcen

Yun, Chawon

Yun, Jonghwa

Yurac, Ratko

Yusof, Abdul Halim

Zaborovskii, Nikita

Zakrzewski, Jack

Zamorano, Juan José

Zanirato, Andrea

Zarei, Mohammad Reza

Zavanone, Mario

Zdun-Ryżewska, Agata

Zeman, Jaroslav

Zeman, Petr

Zendehdel, Kazem

Zeng, Changchun

Zeng, Yan

Zenga, Francesco

Zhang, Cong

Zhang, Guowang

Zhang, Hao

Zhang, Jianguo

Zhang, JianXiang

Zhang, Min

Zhang, Shuo 
Zhang, Xuesong

Zhang, Yanbin

Zhang, Yang

Zhang, Yilong

Zhang, YinShun

Zhang, Yue
Zhang, Zhiqi

Zhang, Zhishan

Zhao, Liang

Zhao, Xinghua

Zhao, Zhigang

Zheng, Guoquan
Zhong, Junlong

Zhong, Woquan

Zhou, Bangjian

Zhou, Chunguang

Zhou, Fang

Zhou, Haitao
Zhu, Ce

Zhu, Mary

Zhu, Wenyi

Zhu, Zezhang

Zubkov, Micaella

\section{Authors/Speakers with Conflict of Interest}

\section{LEGEND}

OI Royalties from a company or supplier

02 Speakers bureau/paid presentations for a company or supplier

03a Paid employee for a company or supplier

03b Paid consultant for a company of supplier

03c Unpaid consultant for a company or supplier

04 Stock or stock options in a company or supplier

05 Research support from a company or supplier as a PI

06 Other financial or material support from a company or supplier

07 Medical/Orthopaedic publications editorial/governing board

08 Board member/committee appointments for a society

09 Other support (please specify)

Acaroglu, Emre

Adhikari, Prashant

Agarwal, Anand

Alanay, Ahmet

Allain, Jérome Alvarado, Fernando

Ames, Christopher

Antoniou, John Ardura, Francisco

Arnold, Paul

Asghar, Jahangir

Bae, Junseok

Banagan, Kelley

Benneker, Lorin M.

Benton, Adam

Benzel, Edward

Bernal, Luis

Berven, Sigurd
02: AO Spine, Medtronic, Stryker, Zimmer Biomet, AO Spine,Medtronic, Stryker Spine, Zimmer Biomet 04: IncredX, IncredX (self-managed), 05: Depur Synthes,Medtronic, Stryker, DePuy Synthes, Medtronic, Stryker, DePuy Synthes, Medtronic, Stryker Spine, 08: Advisory Board: AO Spine, AO Spine,

05: Medtronic, Medtronics 09: Grants/Research Support: Medtronic,

0I: Endosphere, Butterfly,Spinal Balance, OsteoNovus 03a: OsteoNovus, Spinal Balance, 03b: OsteoNovus, Spinal Balance,04: Spinal Balance, OsteoNovus, Intellisenze, 05: Paradigm Spine, Joimax, Element Orthopedics,BONE LLC., 06: Paradigm Spine, Joimax, Element Orthopedics, Endosphere Spine, Butterfly Spine, Intellisenze, BONE LLC., NSF, Third Frontier Program, ODSA, 09: NSF, Third Frontier Program, ODSA,

03a: Depuy Synthes, Stryker, Medtronic 03b: Depuy Synthes, Stryker, Medtronic 03c: DePuy Synthes, Medtronic, Stryker, 05: DePuy Synthes,

0I: LDR/ZIMMER 03b: LDR/ZIMMER

08: AOspine Latinoamérica.

0 I: Aesculap, Biomet,Fish and Richardson PC, Styker Spine, Zimmer Biomet Spine 03b: DePuy Synthes, Medtronic, Stryker Spine,DePuy Synthes, Medtronic, Styker Spine,Depuy, Medtronic, Stryker, 04: Baxano, Doctor's Research Group,05: Depuy Synthes Spine, 06: Fish \& Richardson, P.C., Stryker Spine, Zimmer Biomet Spine,

04: Trepso Therapeutics Inc.

03b: VEXIM MEDCOMTECH

03b: Medtronic Sofamore Danek Stryker Spine Fziomed,Medtronic Sofamore Danek Stryker Spine

Fziomed,Medtronic Sofamore Danek Stryker Spine Fziomed 04: Zplasty, 05: AOSpine International,

03b: Globus Medical

03c: Joimax $\mathrm{GmbH}$, Cousin biotech,Joimax $\mathrm{GmbH}$, Cousion Biotech 08: Board member of Korean spinal deformity research society,Korean spinal deformity research society,

03a: K2M 05: Orthofix,

03b: Icotec AG, Altstaetten, Switzerland

04: Infinity Health

0 I: Elsevier Pub., Thieme Pub.,Elsevier, Thieme publishers,Prevent Biometrics, Inc. OrthoMEMS, Inc. Axiomed Spine Corporation 02: Multiple,03b: Axiomed,Turning Point Biotechnology Axiomed Spine Corporation, 03c: Axiomed, 04: Axiomed, Deputy, Orthomems, Turningpoint,Axiomed, Depuy, Orthomems, Turning Point, 05: Rawlings, 06: Axiomed Spine Corporation Prevent Biometrics, Inc., 07: World Neurosurgery - Editor in Chief, 09: OREF grant, Multiple trips/travel received,

04: RSTT GmbH

0I: Medtronic Stryker,Medtronic 02: Medtronic, Globus Medical, RTI, Stryker, 03b: Medtronic Stryker Globus Titan,04: Providence Medical, 07: Journal of Bone and Joint Surgery; Journal of Neurosurgery; Orthopaedics Today; Spine Deformity Journal; Spine, 08: Scoliosis Research Society; AO Spine; Bone and Joint Decade, 09: Research Support: AO spine; Omega; OREF, 
Bess, Shay

Bettegowda, Chetan

Bilsky, Mark

Birkenmaier, Christof

Bleys, Ronald

Blumenthal, Scott

Boachie-Adjei, Oheneba

Bonassar, Lawrence

Boriani, Stefano

Breakwell, Lee

Brodke, Darrel S.

Buchowski, Jacob

Bucklen, Brandon

Burch, Shane

Burton, Douglas

Bydon, Ali

Cabiol Belmonte, Jose

Cahill, Patrick

Caiazzo, Francesco

Cannestra, Andrew

Caridi, John

Carreon, Leah

Casimiro, Miguel

Castelein, René

Cheng, Joseph

Cheung, Berry

Cho, Samuel

Cho, Woojin

CHOI, DAE-JUNG

Choi, David

Choma, Theodore J.

Christie, Sean

Chu, L H

Clarke, Michelle

Coe, Jeffrey

Cofano, Fabio

Cole, Ashley

Crutcher, Clifford

Dahl, Benny

de Kleuver, Marinus

Deutsch, Harel

Deviren, Vedat

Dhakal, Gaurav Raj

Disselhorst-Klug,

Catherine

Domingo-Sabat,

Montse

Elowitz, Eric

Erickson, Mark
0 I: Innovasis, K2M, NuVasive, 02: K2M,03b: Allosource, K2M,Allosource, K2M, 05: DePuy Synthes, K2M, Medtronic, NuVasive, Stryker Spine, Zimmer Biomet, 06: Innovasis, K2M, NuVasive, 08: Allosource,

05: AOSpine International

0 I: Globus 02: Varian,03b: DePuy/Synthes, Globus, and Varian,

04: EOS

06: Galvani Bioelectronics

02: Aesculap, LDR, Vertiflex 04: Fziomed, Vertiflex, Centinel Spine, 06: Scientific Advisory Board: Aesculap, Fziomed, Vertiflex, 07: Europ Spine J, 08: NASS Chair, Section on Motion Preservation,

02: K2M, WEIGAO 03b: K2M, WEIGAO, 04: K2M,05: K2M, 08: K2M,

03b: Fidia Pharmaceuticals, Histogenics, Inc., and 3DBio Corp. 05: Histogenics, Inc.,

02: AOSpine International

08: British Orthopaedic Association Council Member

0 I: Amedica, DePuy Synthes, and Medtronic 03b: Amedica, DePuy Synthes,04: Amedica,

02: Globus Medical 03b: Globus Medical, K2M, Medtronic, 05: AOSpine North America, OMeGA, 06: Globus

Medical, K2M, Wolters Kluwer Health, Inc.,

03a: Globus Medical Inc. 04: Globus Medical Inc.

03b: Medtronic 05: Globus AOSpine Nuvasive,

03b: DePuy Synthes 05: DePuy Synthes,06: DePuy Synthes,

0 I: Research grant from DePuy Spine and serves on the clinical advisory board of Medlmmune, LLC.

03b: Nuvasive

03b: Biogen

02: Nuvasive 03b: Nuvasive,

OI: Nuvasive, RTI 03b: Nuvasive, RTI, Alliance Spine, Mazor Robotics, Spinal Elements,05: Mazor Robotics,

03b: Zimmer Biomet

03b: AO Spine 07: Spine, spine Journal, Spine Deformity, 08: Scoliosis Research Society, University of Louisville Institutional Review Board,

02: Ulrich Medical Synthes

05: K2M Research Grant,Received an unrestricted K2M research grant

08: Editorial Board, JNS Spine Board of Directors, NASS

03a: Avalon SpineCare (HK)

03b: Zimmer Biomet, Globus, Medtronic, 05: Zimmer Biomet, 08: SRS, CSRS, NASS, AOSpine, AAOS, AOA,

03c: Globus, DIO 07: Editorial Board, Global Spine Journal (20I4-present) Editorial Board, Asian Spine Journal (20I5present) Editorial Board, Montefiore Journal of Musculoskeletal (2016-present), 08: Educational Committee, Scoliosis Research Society, August $2014 \sim$ CME Committee, Scoliosis Research Society, August 2015 International Education Committee, North American Spine Society, June 2017

03c: BMK C\&S Medical Synus Medical, 05: BMK C\&S Medical Synus Medical, 08: Minimally Invasive Spine Surgery of Korean Orthopaedic Spine Society,

05: DePuy Synthes

04: Gentis Inc. 05: DePuy Spine, Stryker Spine, 08: Scoliosis Research Society, AOSpine North America,

05: Medtronic 06: Rick Hansen Institute,

03a: Avalon SpineCare (HK)

05: AOSpine International

03b: Benvenue Medical, Nuvasive, Providence Medical Technology 04: Alphatec, Phygen,05: Benvenue Medical, Nuvasive, 08: Committee Membership: SRS, CSRS, LSRS Board Membership: California Orthopaedic Association, 0 I: $\mathrm{NCH}$ Molinette

08: Committee of British Scoliosis Society Committee of UK Spinal Societies Board Spinal Chair of the Orthopaedic Expert Working Group advising NHS Chair of Spinal Services CRG, NHS England Clinical Lead for the NHS England Improving Spinal Care Project

09: Corelink, Stryker- travel grants to attend resident educational courses

05: Institutional grant from K2M and Medtronic,

02: DePuy Synthes, Medtronic 08: AO Spine, Scoliosis Research Society,Board member AOSpine KF Deformity and

Scoliosis Research Society, 09: Netherlands health insurance innovation fund (grant/research support),

03b: Baxter Healthcare Corporation and RTI Surgical

0I: Nuvasive 03b: Nuvasive,05: Nuvasive,

09: Research Project of AO Spine Research Commission Mentor-Mentee Program

05: Otto Bock Healthcare

05: DePuy Synthes

03b: NuVasive

02: Zimmer Biomet 
Fehlings, Michael

Fiol Busquets, Bartolomé Fiorentin, Joana Fischgrund, Jeffrey Fisher, Charles

Foley, Kevin

Frank, Steven M.

Gandhi, Anup

Garg, Sumeet

Gehrchen, Martin

Gelb, Daniel

Gennari, Antoine

Ghag, Ravi

Ghosh, Peter

Glassman, Steven

Goel, Vijay K.

Gokaslan, Ziya

Goldschlager, Tony Goldstein, Christina L. Good, Christopher Goodwin, C. Rory

Greeley, Samantha Guasque, Joana Guo, Chang Ming

Gupta, Munish Guyer, Richard

Harris, Jonathan Härtl, Roger Hostin, Richard Hsu, Erin

Hsu, Victor Hsu, Wellington

Ikeme, Shelly Inzana, Jason Jacobsen, Michael Kjaer Jadik, Senol Kawaguchi, Yoshiharu Kellersmann, Angelika Kelly, Michael Khanna, Nitin Kim, Han Jo Kim, Jin-Sung "Luke”
02: Pfizer Zimmer Biomed, 03b: Pfizer - Consulting agreement (speaker) Zimmer Biomed - Consulting agreement (speaker) In Vivo Therapeutics - consulting agreement 03b: Nuvasive

07: Joana Guasque 08: Joana Guasque 09: Joana Guasque,

09: Clinical Research - Relievant Medsystems

0I: Medtronic and Nuvasive, research support from OREF, AOSpine, and Medtronic 03b: Medtronic, Nuvasive, 05: AOSpine International, OREF grant paid to institution 06: AOSpine and Medtronic support for fellowship paid to institution, 09: Orthopaedic Research and Education Foundation (Grant for this project) Medtronic, AOSpine, Johnson \& Johnson (Fellowship support-paid to your institution),

0 I: Medtronic 03b: Medtronic, 04: Discgenics, Medtronic, NuVasive, SpineWave, TrueVision, Vertera, 08: Discgenics, TrueVision,

03b: Consulting relationships with Haemonetics and Medtronic, Consulting relationships with Haemonetics and Medtronic.

03a: Zimmer Biomet

03b: Medtronic, Mighty Oak Medical 06: Decision Support in Medicine,

05: Institutional grant from K2M and Medtronic

01: Depuy-Synthes Spine Globus Medical 02: Depuy-Synthes Spine,04: Advanced Spinal Intellectual Property,

05: LDR/ZIMMER

05: DePuySynthes 08: E-text committee of Scoliosis Research Society,

0I: Mesoblast consultant 03b: Mesoblast,

01: Medtronic 03a: Employee of Norton Healthcare, 03b: Medtronic, 05: Norton Healthcare,08: Past President Scoliosis Research Society - Unpaid position,

0I: Spinal Balance, OsteoNovus, Globus, Endosphere, Butterfly 04: Spinal Balance, OsteoNovus, Intellisenze, 05: Turning Point, DePuy, SI Bone, Apex/Spine/Medyssey, Spine Soft Fusion, Spinal Elements, AO Foundation/FORE, K2M, NIH, NSF, Third Frontier Program, ODSA, OsteoNovus, Spinal Balance, Spine Scoliosis LLc, FDA, Spider Spine LLc, 4 WEB 09: NIH, NSF,

OI: Stock ownership in US Spine and Spinal Kinetics, consulting, speaking and teaching for the AO Foundation and Research support from Depuy, NREF, AOSpine and AO North America 04: Spinal Kinetics 05: AOSpine International 09: AOSpine (Travel and Grants),

01: Mesoblast consultant 03b: Mesoblast,

05: MU Coulter Translational Partnership 08: AOSpine North America, North American Spine Society,

05: Mazor Robotics

05: UNCF Merck Postdoctoral Fellow and has received an award from the Burroughs Wellcome Fund and the Johns Hopkins Neurosurgery Pain Research Institute. 09: UNCF Merck Postdoctoral Fellow and has received an award from the Burroughs Wellcome Fund and the Johns Hopkins Neurosurgery Pain Research Institute.,

03a: Globus Medical Inc. 04: Globus Medical Inc.,

07: Joana Guasque 08: Joana Guasque,09: Joana Guasque,

02: Eli Lilly: Paid presenter or speaker Nuvasive: Paid presenter or speaker 08: Singapore Orthopaedic Association: Board or committee member,

02: DePuy, 03b: DePuy, 04: Johnson \& Johnson, Proctor \& Gamble,

01: Alphatec 02: Synthes, Aesculap, MiMedx,03b: DePuy Spine,04: Spinal Motion, Flexuspine, Spinal Kinetics, 07: Editorial board of Spine and Spine J,08: member of Motion Preservation Section at NASS,09: Scientific Advisory Board: K2M, Flexuspine, Spinal Kinetics, Nanovis, MiMedx, Crocker Technologies,

03a: Globus Medical Inc. 04: Globus Medical Inc.,

0I: Lanx 03b: DePuy Synthes, Brainlab and Ulrich,

03b: DePuy Synthes

0I: Stryker 02: AONA,03b: Stryker, Bacterin, Graftys, Globus, AONA, SI Bone, Relievant, Ceramtec, Medtronic Sofamor Danek, Bioventus, LifeNet, RTI,05: Medtronic,

05: Mazor Robotics

0I: Stryker 02: AONA, 03b: Stryker, Bacterin, Graftys, Globus, AONA, SI Bone, Relievant, Ceramtec, Medtronic Sofamor Danek, Bioventus, LifeNet, RTI, 05: Medtronic, 09: Clinical Research - Relievant Medsystems,

03a: Baxter Healthcare Corporation

03a: Zimmer Biomet

0I: $30.000 \mathrm{kr}$ for statistical assistance

03b: Vexim, Intrinsic Therapeutics

07: Journal of Orthopaedic Surgery, Spine Surgery Related Research 08: AO Spine

07: Ehab Shiban 08: Ehab Shiban, 09: Ehab Shiban,

05: DePuy Synthes,

03b: Baxter Healthcare Corporation, Nuvasive, Globus, Edge Surgical, Alpheon, and Casetabs

03b: K2M, Zimmer Biomet 06: K2M, Zimmer Biomet,08: AOSpine,

2: RIWOspine, Elliquence 


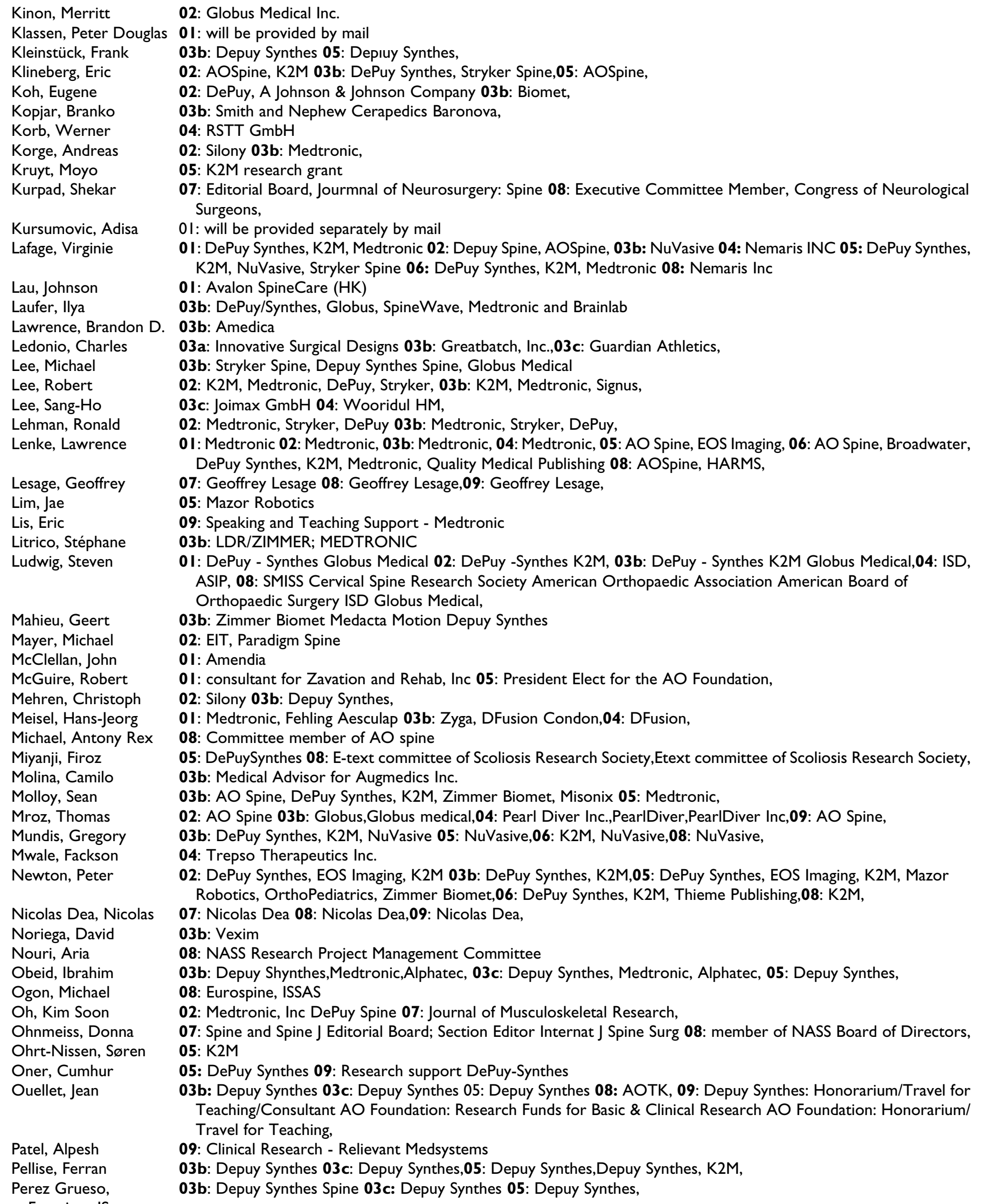

Francisco JS 
Pittman, Jason $\quad$ 03b: DePuy Synthese

Rahimi-Movaghar, Vafa 03a: Sina Trauma and Surgery Research Center, Tehran University of Medical Sciences

Raus, Laura

Reilly, Christopher

Rhines, Laurence

Rhyne, Alfred

Riew, K.Daniel

Rodriguez, Andres

Russo, Scott

Sacks, Justin

Sahgal, Arjun

Samdani, Amer

Santaguida, Carlo

Sarwahi, Vishal

Sasso, Rick

Sato, Shigenogu

Savage, Jason

Schnake, Klaus John

Schroerlucke, Samuel

Schuster, James

Schwab, Frank

01: BBraun, Royalties for the patent of the product Kairison

05: DePuySynthes 08: E-text committee of Scoliosis Research Society, Etext committee of Scoliosis Research Society,

03b: Globus, Stryker, Globus, Stryker, 05: AOSpine International 09: AOSpine (Travel),

09: Clinical Research - Relievant Medsystems

01: Biomet, Medtronic 04: Osprey, Expanding Orthopedics, Spinal Kinetics, Amedica, Nexgen Spine, Vertiflex,

Benvenue, Paradigm Spine, PSD, Spineology, 07: Global Spine J, Spine, Neurosurgery, Spine this Week, Clinics in

Orthopaedics, Spine Surgery Today, 08: AO Spine,

02: Pfizer

03b: Medtronic

0I: Consulting relationship with LifeCell.

05: AOSpine International

03b: DePuy Synthes, Ethicon, Globus Medical, Misonix, Stryker Spine, Zimmer Biomet

02: Medtronic and Stryker 03b: Stryker,05: CSL Behring,07: Deputy editor of Clinical Spine Surgery Journal,

03b: Precision Spine, Medtronic 06: Precision Spine,

09: Clinical Research - Relievant Medsystems

01: Orthopaedic Spine Surgeon 02: Orthopaedic Spine Surgeon,03a: Orthopaedic Spine Surgeon,03b: Orthopaedic Spine Surgeon,03c: Orthopaedic Spine Surgeon, 04: Orthopaedic Spine Surgeon,06: Orthopaedic Spine Surgeon,

07: Kawakami Mamoru,08: Kawakami Mamoru,09: Kawakami Mamoru,

03b: Stryker Corporation

05: Otto Bock Healthcare 08: AOSpine International,

05: Mazor Robotics

05: AOSpine International

0I: K2M, Medtronic, NuVasive, Zimmer Biomet 02: NuVasive,03b: K2M, Medicrea, Medtronic, NuVasive, Zimmer Biomet,04: Nemaris INC,05: DePuy Synthes, K2M, NuVasive, Stryker Spine,DePuy Synthes, K2M, Stryker Spine, 06: K2M, Medtronic, NuVasive, Zimmer Biomet,

Sciubba, Daniel $\quad$ OI: has consulting relationships with Medtronic, Globus, DePuy, and Orthofix 03b: Consulting relationships with

Medtronic, Globus, DePuy, and Stryker, Orthofix, Nuvasive K2M 05: AOSpine International,

Seevinck, Peter

Serhan, Hassan

Shaffrey, Christopher

04: MRlguidance BV 05: Xilloc BV, MRlguidance BV, ZimmerBiomet,

0 I: DePuy SYNTHES Spine, J\&J stock holder

0 I: Medtronic, NuVasive, Zimmer Biomet 03b: K2M, Medtronic, NuVasive, Stryker Spine,K2M, Medtronic, NuVasive,

Styker Spine,04: NuVasive,06: Medtronic, NuVasive, Zimmer Biomet,

Shah, Suken

Shufflebarger, Harry

Siepe, Christoph

Smith, Justin

02: DePuy Synthes 03b: DePuy Synthes, NuVasive,05: DePuy Synthes,06: K2M,08: DePuy Synthes,

02: DePuy Synthes, K2M 03b: DePuy Synthes, K2M,06: DePuy Synthes, K2M,

02: RiWo Spine, Silony 03b: Depuy Synthes,

O I: Zimmer Biomet, K2M 03b: NuVasive, Zimmer Biomet,05: AO Spine, DePuy Synthes,AOSpine, DePuy Synthes,

06: K2M, Zimmer Biomet,

Spiker, William Ryan

Sponseller, Paul

Steinmetz, Michael

Sucato, Daniel

Sweeney, Thomas

Thomé, Claudius

Tsang, Parker

Vajkoczy, Peter

Van de Giessen,

Martijn

van Stralen, Marijn

Varga, Peter Pal

Versteeg, Anne

Vialle, Emiliano

03b: Amedica, NEXXT, and Nexus

03b: DePuy Synthes, Globus Medical 05: DePuy Synthes,06: DePuy Synthes, Globus Medical, JBJS,

0 I: Biomet Spine,Biomet, Inc. 03b: Biomet,Biomet spine,Globus Medical, Inc. Stryker Corporation,

06: Globus Medical

05: Mazor Robotics

02: Aesculap, DePuy Synthes, Brainlab, Medtronic, Intrinsic Therapeutics, TETEC, Ulrich, Signus Medizintechnik

03a: Avalon SpineCare(HK)

09: Clinical Research - Relievant Medsystems, Inc.

03a: Philips

03b: MRlguidance BV 04: MRIguidance BV,

02: AOSpine International 05: AOSpine International,

03b: AOSpine International 05: AO Spine,

0I: Clin col vertebral 02: Clin col vertebral,03a: Clin col vertebral,03b: Clin col vertebral,03c: Clin col vertebral,

04: Clin col vertebral,06: Clin col vertebral,07: Emiliano Vialle,08: Emiliano Vialle,09: Emiliano Vialle,

Vila-Casademunt, Alba 05: Depuy Synthes

Villalobos, Hunaldo 05: Mazor Robotics

Wang, Jeffrey

0 I: Aesculap, Biomet, Amedica, Seaspine, Synthes 04: Stocks/Private investments: Fziomed;Promethean Spine, Paradigm spine, Benevenue, NexGen, Vertiflex, electrocore, surgitech, expanding orthopaedics, osprey, bone biologics, curative biosciences, pearldiver,08: North American Spine Society (Second Vice President), North

American Spine Foundation (non-financial), Cervical Spine Research Society (Travel expenses), AO Spine/AO

Foundation (honorariums for board position),09: Fellowship Support: AO Foundation (spine fellowship funding paid to institution)

Wang, Michael $\quad$ 05: Mazor Robotics 
Wang, Wenhai $\quad 03$ a: Globus Medical Inc. 04: Globus Medical Inc.,

Wasserberg, Jonathan $\quad$ II: Dept of Neurosurgery, Queen Elizabeth Ho 02: Dept of Neurosurgery, Queen Elizabeth Ho,03a: Dept of Neurosurgery, Queen Elizabeth Ho,03b: Dept of Neurosurgery, Queen Elizabeth Ho,03c: Dept of Neurosurgery, Queen Elizabeth Ho,04: Dept of Neurosurgery, Queen Elizabeth Ho,06: Dept of Neurosurgery, Queen Elizabeth Ho,07: Jonathan Wasserberg,08: Jonathan Wasserberg,09: Jonathan Wasserberg,

Weber, Michael 05: AOSpine International

Weidert, Simon

03b: Brainlab 04: Medability GmbH, Mecuris GmbH, M3i GmbH,05: Ziehm Imaging GmbH, Brainlab AG,07: Dr. med. Simon Weidert,

Witham, Timothy

Yamada, Yoshiya

Yang, Victor

Yassari, Reza

Yoon, S. Tim

Youssef, Jim

Zahrawi, Faissal

Zavatsky, Joseph

Zebala, Lukas

Zigler, Jack

OI: Research grant from Eli Lilly and Company, and non-study-related research from the Gordon and Marilyn Macklin Foundation

03b: Varian Medical Systems 08: Chordoma Foundation,

03c: Chief Scientific Officer for 7D Surgical Inc.

07: Section Editor, World Neurosurgery

0 I: Stryker Spine, Meditech Spine, 04: Medyssey, Phygen, Alphatec, Meditech,

0 I: Amedica, Osprey Biomechanical 03b: Health Trust, Nuvasive, SeaSpine,,04: Paradigm Spine, Benvenue,

05: Mazor Robotics

0 I: Zimmer Biomet 03b: DePuy Synthes Spine, Stryker, Zimmer Biomet, Vivex, Sentio 04: Vivex, Sentio,05: Zimmer Biomet, DePuy Synthes Spine, Stryker, Vivex, Sentio,

02: Broadwater

01: Zimmer 02: Synthes Spine, MiMedx, 03b: DePuy Spine, Aesculap, Medtronic Spine, Orthofix, Simplify Medical, FloSpine, 04: Expanding Orthopedics, Safe Orthopedics,07: Section Editor for International J Spine Surgery,

08: Board of Directors member for ISASS, 09: Scientific Advisory Board: SafeOrthopedics,

Zygourakis, Corinna

09: Received travel expenses to attend fellow spine education course from Depuy 